\title{
HIGH-POWER ELECTRON BEAM INJECTORS FOR 100 KW FREE-ELECTRON LASERS ${ }^{\dagger}$
}

\author{
A. Todd, H. Bluem, V. Christina, M. Cole, J. Rathke, T. Schultheiss, \\ Advanced Energy Systems, P.O. Box 7455, Princeton, New Jersey 08543-7455, USA
}

I. Campisi, E. Daly, D. Douglas, G. Neil, J. Preble, R. Rimmer, C. Rode, J. Sekutowicz, T. Whitlatch, M. Wiseman, Thomas Jefferson National Accelerator Facility, Newport News, VA 23606, USA

P. Colestock, J. P. Kelley, S. Kurennoy, D. Nguyen, S. Russell, D. Schrage, R. Wood, L. Young, Los Alamos National Laboratory, Los Alamos, NM 87545, USA

\section{Abstract}

A key technology issue on the path to high-power FEL operation is the demonstration of reliable, highbrightness, high-power injector operation. We describe two ongoing programs to produce $100 \mathrm{~mA}$ injectors as drivers for $100 \mathrm{~kW}$ free-electron lasers. In one approach, in collaboration with the Thomas Jefferson National Accelerator Facility, we are fabricating a $750 \mathrm{MHz}$ superconducting RF cryomodule that will be integrated with a room-temperature DC photocathode gun [1] and tested at the Laboratory. In the other approach, in collaboration with Los Alamos National Laboratory, a high-current $700 \mathrm{MHz}$, normal-conducting, RF photoinjector $[2,3]$ is being designed and will undergo thermal management testing at the Laboratory. We describe the design, the projected performance and the status of both injectors.

\section{INTRODUCTION}

High-power free-electron lasers (FEL) have been proposed for both commercial and defence applications. Commercial FEL material processing applications [4] have been proven using conventional lasers and patented, but their market insertion has been impeded by the lack of suitable, economic light sources. $100 \mathrm{~kW}$ IR and $10 \mathrm{~kW}$ UV FEL devices producing radiation for a lifecycle cost of less than one cent per delivered $\mathrm{kJ}$ in the IR and a few cents per $\mathrm{kJ}$ in the UV have tremendous market potential. However, in order to achieve these radiation cost targets, high-current, low emittance electron beam injectors must be developed to drive the energy-recovery-linacs (ERL) [5] that can deliver the required wall-plug electrical efficiency needed to approach these radiation costs [6].

Advanced Energy Systems (AES) has been collaborating with Los Alamos National Laboratory and the Thomas Jefferson National Accelerator Facility (JLAB) on the development of two distinct $100 \mathrm{~mA}$ highbrightness electron injector systems. The Los Alamos device is a normal-conducting, water-cooled $700 \mathrm{MHz} \mathrm{RF}$

\footnotetext{
Supported by the US Department of Defense Missile Defense Agency and Naval Sea Systems Command PMS-405 under Strategic Missile and Defense Command contract no. DASG60-02-C-0003 and by the Joint Technology Office under Envisioneering contract no. S02-08. Jefferson Laboratory personnel are supported under US Department of Energy contract no. DE-AC05-84ER40150 and Advanced Energy Systems CRADA SURA-2002S003. Los Alamos personnel are supported by the Joint Technology Office under a Naval Sea Systems Command contract.
}

photo-injector while the JLAB device closely couples a DC gun and a $750 \mathrm{MHz}$ superconducting RF (SRF) accelerator that utilizes single-cell cavities to handle the high RF input power. The injectors described below, given performance requirements of $>100 \mathrm{~mA},<1 \%$ energy spread, $<10$ microns transverse and $<100-200$ $\mathrm{keV}$-psec longitudinal rms normalized emittance will be capable of driving $100 \mathrm{~kW}$ and higher power IR FEL devices.

\section{GUN AND SRF INJECTOR}

The JLAB $10 \mathrm{~kW}$ IR FEL will utilize a DC gun and CEBAF "quarter cavity" linac to deliver $10 \mathrm{~mA}$ to the recirculating arc. For the $100 \mathrm{~mA}$ injector needed to drive a $100 \mathrm{~kW}$ FEL, the electron beam power approaches one megawatt and it is desirable to utilize single cell cavities. To provide high-order mode (HOM) and beam breakup (BBU) instability headroom, the frequency has been reduced to the $750 \mathrm{MHz}$ subharmonic of the present FEL.

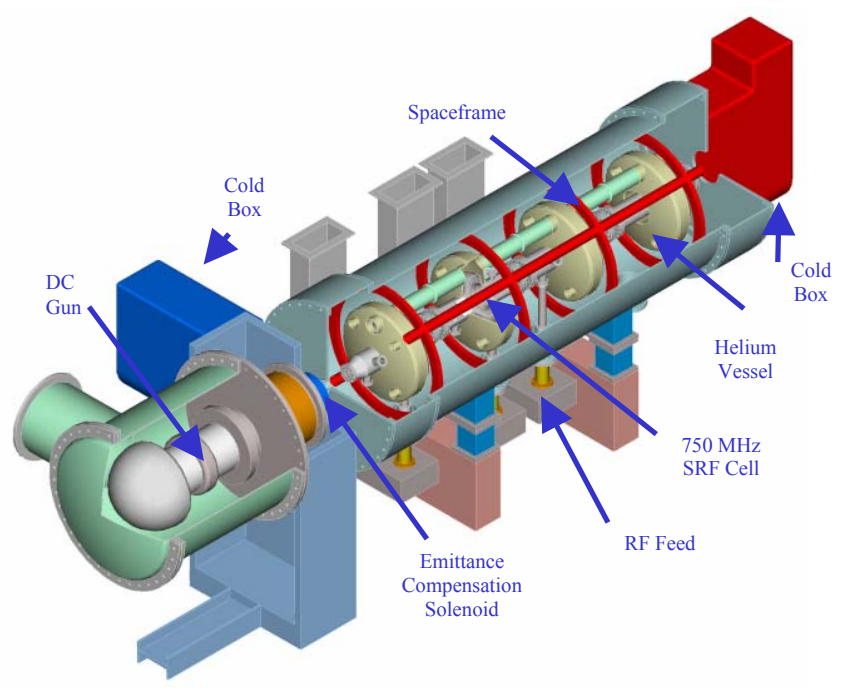

Figure 1: Isometric diagram of the $750 \mathrm{MHz} \mathrm{DC}$ gun and single-cell $100 \mathrm{~mA} \mathrm{SRF}$ injector.

AES has completed the physics design of the three cell $750 \mathrm{MHz}, 7 \mathrm{MeV}$ SRF injector linac, utilizing transverse emittance compensation [7], that is shown in Fig. 1. The fourth upstream cell location is presently used for helium inventory and does not contain an accelerating cell. With a $500 \mathrm{kV}$ DC gun, a pulse repetition frequency (PRF) of 
$750 \mathrm{MHz}$ and $133 \mathrm{pC}$ bunches, our analysis shows that this injector delivers a $7 \mathrm{MeV}, 100 \mathrm{~mA}$ beam with a transverse normalized rms emittance of 1.2 microns, a longitudinal emittance of $44 \mathrm{keV}-\mathrm{psec}$ and an energy spread of $0.5 \%$. As noted, this will meet the beam requirements at the wiggler for a $100 \mathrm{~kW}$ IR FEL.

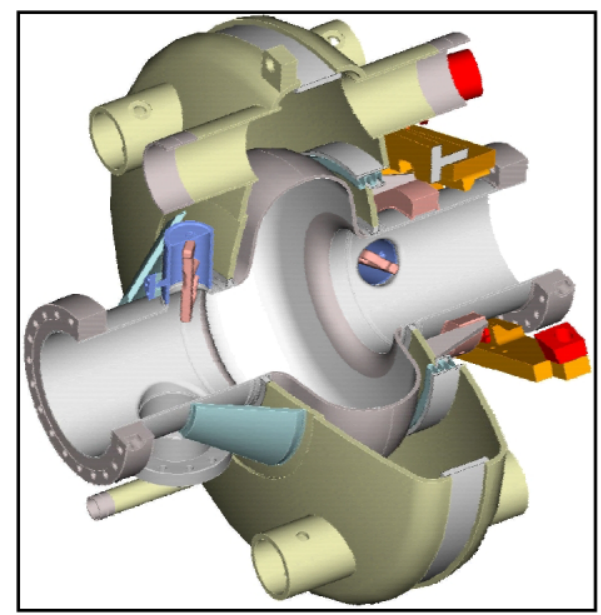

Figure 2: SRF injector cell

The key engineering issues with the injector are the achievement of $500 \mathrm{kV}$ from the upgraded JLAB DC gun [8] and the impact of the high RF power level in the SRF accelerator. The present JLAB DC gun on the $10 \mathrm{~kW}$ FEL is undergoing conditioning to the $500 \mathrm{kV}$ level. We have performed RF, thermal and stress analysis on the SRF cells that indicates the device should be capable of CW $100 \mathrm{~mA}$ operation and removing the expected HOM power load. A single cell cavity is illustrated in Fig.2.

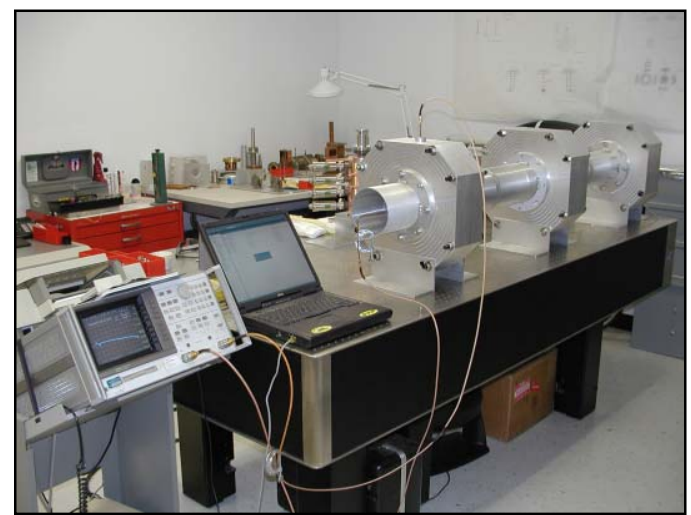

Figure 3: SRF injector cold model undergoing testing

This injector is now in the midst of the fabrication cycle and all components are scheduled for delivery to JLAB by late 2003. Cold modelling to finalize the RF coupler and HOM outcoupler details is in progress at AES as shown in Fig. 3. The device is " $100 \mathrm{~mA}$-capable" with the exception of the three SNS-style RF power couplers [9]. $500 \mathrm{~kW}$ replacement couplers are presently being developed in a parallel program, but because the funding for the RF and DC power supplies needed to drive the device at $100 \mathrm{~mA} \mathrm{CW}$ has not been authorized at this time, initial testing will be at full bunch charge but lower PRF in the JLAB injector test stand. Reduced PRF $1 \mathrm{nC}$ single bunch performance measurements are also planned to examine the viability of extrapolating this technology to even higher power levels. Should the device meet the specified performance goals, it has been proposed that it be installed on the existing JLAB FEL as shown in Fig. 4 as the front end of a $100 \mathrm{~kW}$ upgrade to that device.

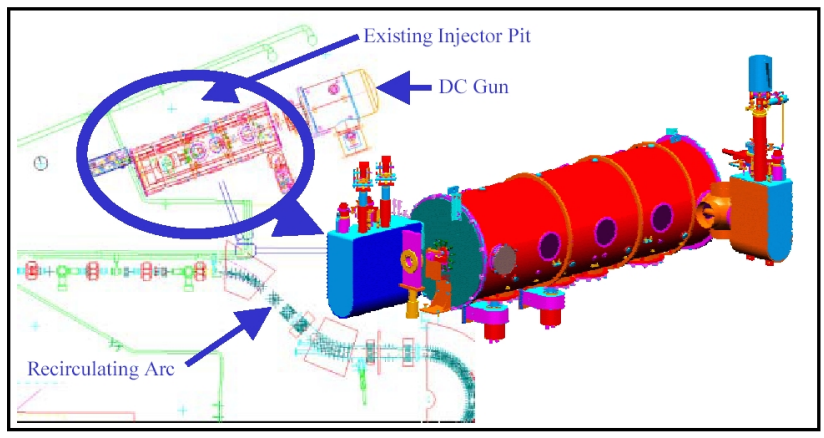

Figure 4: Injector accelerator cold mass in proposed installed position at the JLAB IR FEL.

\section{RF PHOTO-INJECTOR}

The Los Alamos $100 \mathrm{~mA}$ injector concept begins with a 2.5 cell water-cooled, RF photo-injector with a focusing and bucking solenoid. This is followed by a 4 cell normal-conducting booster linear accelerator. The accelerating field in the gun is held to $7 \mathrm{MV} / \mathrm{m}$ and reduced in the booster to control resistive power losses. The solenoid magnetic and gun accelerating fields are shown on Fig. 5. The end cavity on the right is a nonresonant vacuum pumping cavity.

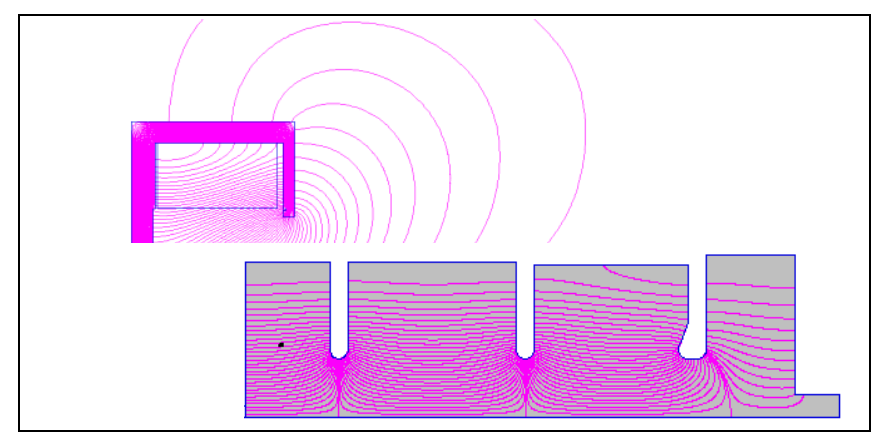

Figure 5: Focussing and bucking solenoids with their magnetic fields and the electric fields in the accelerator for the normal-conducting injector.

The physics and conceptual engineering design of this injector has been performed by Los Alamos. With a bunch charge of $3 \mathrm{nC}$, the design achieves a transverse rms normalized emittance of 7 microns, a longitudinal emittance of $200 \mathrm{keV}$-psec and an energy spread of less than $1 \%$. The energy at the end of the gun is $2.7 \mathrm{MeV}$ rising to $5.5 \mathrm{MeV}$ after the booster. At a PRF of 33.3 $\mathrm{MHz}$, the injector delivers $100 \mathrm{~mA}$, which value increases to greater than $1 \mathrm{~A}$ at a PRF of $350 \mathrm{MHz}$.

The key issue for this normal-conducting copper injector, which is illustrated in Fig. 6, is the thermal management of the resistive losses at the high accelerating gradient needed to generate high-brightness 


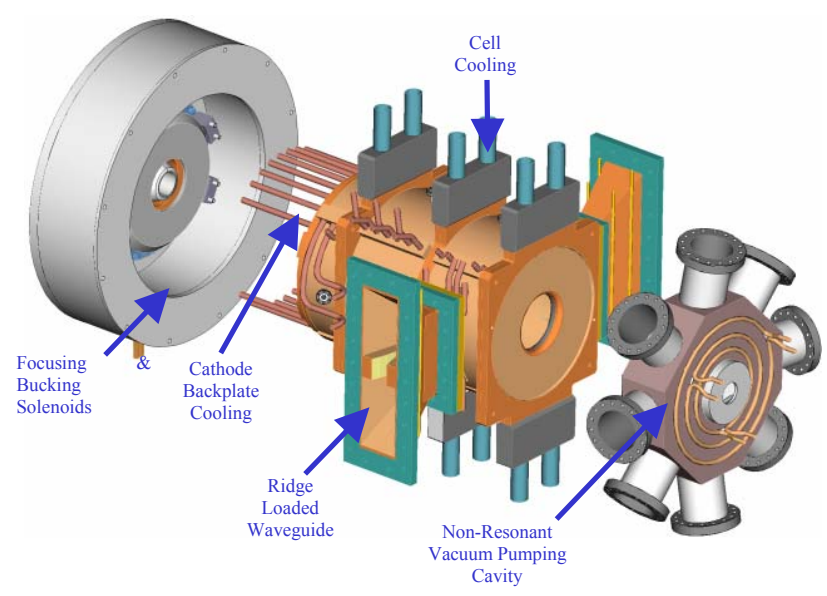

Figure 6: Schematic diagram of the $700 \mathrm{MHz}, 100 \mathrm{~mA}$ photo-injector.

in the electron beam. AES has performed the critical thermal and stress analysis of the device which demonstrates that adequate cooling can be provided at flow rates that do not cause erosion or excessive vibration while maintaining the desired temperature stability. Fig. 7 shows the steady-state thermal distribution where it can be seen that the temperature variation is constrained to less than $30 \mathrm{C}$ throughout the gun. The corresponding stress analysis indicates sufficient margin for the Glidcop gun material as shown in Fig. 8.

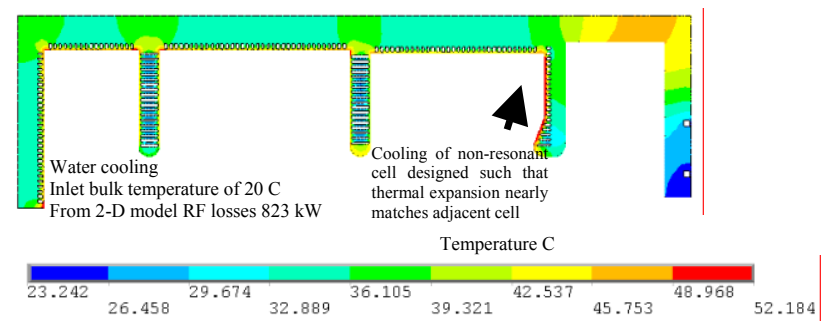

Figure 7: Steady-state thermal distribution in the 2.5 cell photo-injector gun.

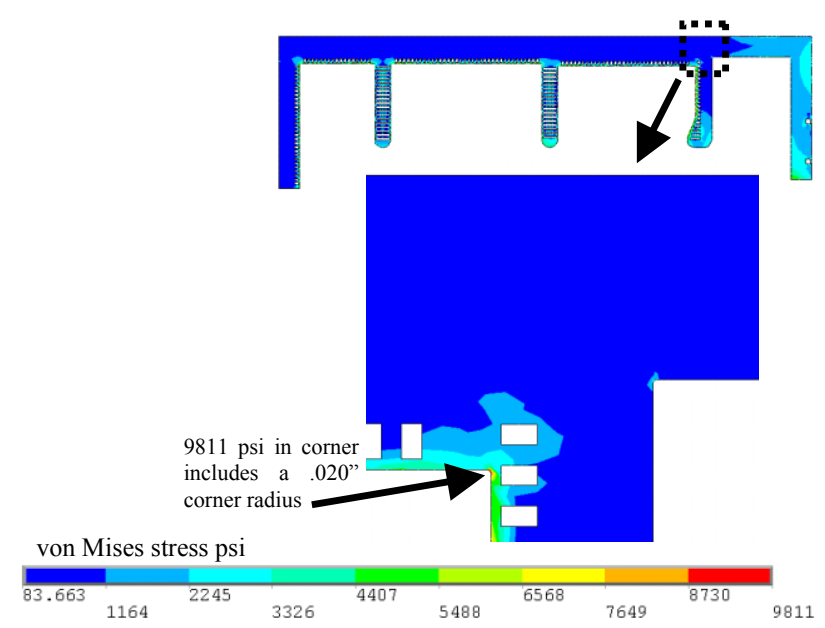

Figure 8: Steady-state thermal stress in the 2.5 cell photo-injector gun showing the peak stress.

This injector has completed the preliminary engineering design phase and long lead item procurement is in process. As with the previous SRF injector concept, it is presently not possible to perform a full power beam test. Consequently, the plan is for AES to deliver the fabricated gun to Los Alamos in May 2004 for thermal management testing. This will demonstrate that the gun can satisfy the engineering operating requirements. The confirmation of the projected physics performance of the injector can then be completed under a follow-on program.

\section{CONCLUSIONS}

The projected performance and fabrication status of two $100 \mathrm{~mA}$ high-brightness electron beam injector concepts were described. The physics designs of both devices, which are intended to drive 100+ kW IR FEL's, meet the requisite performance specifications. The DC Gun and SRF injector device is in fabrication, with component delivery to JLAB scheduled for late 2003. The RF photoinjector device has completed preliminary design, with gun delivery to Los Alamos scheduled for mid 2004. The testing of both injectors is presently planned for 2004 . Because of equipment limitations at both Laboratory sites, neither device can be beam tested at full current at this time. The JLAB test will be at full charge and reduced current while the Los Alamos test will demonstrate CW thermal management without beam. Both injectors are designed to deliver the specified $100 \mathrm{~mA}$ performance under follow-on programs. In particular, the JLAB injector will be used as the front end of a $100 \mathrm{~kW}$ upgrade to the existing FEL if the required performance is demonstrated and the upgrade program is funded.

\section{REFERENCES}

[1] C. K Sinclair, Nucl. Instr. Meth. A318 (1992) 410-414.

[2] J. S. Fraser et al., "Photocathodes in Accelerator Applications," Proceedings of the 1987 Particle Accelerator Conference, IEEE 87CB2387-9 (1987) 1319.

[3] S. Kurennoy et al., "Photocathode RF Cavity Design for High-Power CW FEL," these Proceedings.

[4] http://www.jlab.org/FEL/LPC/applications.html.

[5] G. R. Neil and the Jefferson Laboratory IR FEL team, "Sustained Kilowatt Lasing in a Free-Electron Laser with Same Cell Energy Recovery," Phys. Rev. Lett. 84, (2000) 662-665.

[6] M. J. Kelley, "Materials Processing Research and Development Opportunities with the New Generation of FEL's," SPIE Proc. 3888 (2000) 598-605.

[7] B.E. Carlsten, "Photoelectric Injector Design Code," Proceedings of the 1989 IEEE Particle Accelerator Conference, IEEE 89CB2669-0 (1989) 313.

[8] S. V. Benson et al., "A $10 \mathrm{~kW}$ IRFEL Design for Jefferson Lab," Proceedings of the 2001 IEEE Particle Accelerator Conference, IEEE 01CH37268 (2001) 249.

[9] I. E. Campisi et al., "The Fundamental Power Coupler for the Spallation Neutron Source (SNS) Superconducting Cavities," Proceedings of the 2001 IEEE Particle Accelerator Conference, IEEE 01CH37268 (2001) 1140. 